\title{
The neuroendocrine phenotype, genomic profile and therapeutic sensitivity of GEPNET cell lines
}

Tobias Hofving1, Yvonne Arvidsson', Bilal Almobarak', Linda Inge1, Roswitha Pfragner2, Marta Persson', Göran Stenman', Erik Kristiansson³, Viktor Johanson ${ }^{4}$ and Ola Nilsson ${ }^{1}$

1Sahlgrenska Cancer Center, Department of Pathology and Genetics, Institute of Biomedicine, Sahlgrenska Academy at the University of Gothenburg, Gothenburg, Sweden

2Institute of Pathophysiology and Immunology, Center for Molecular Medicine, Medical University of Graz, Graz, Austria

${ }^{3}$ Department of Mathematical Sciences, Chalmers University of Technology, Gothenburg, Sweden

${ }^{4}$ Department of Surgery, Institute of Clinical Sciences, Sahlgrenska Academy at the University of Gothenburg, Gothenburg, Sweden

Correspondence should be addressed to T Hofving: tobias.hofving@gu.se

The authors and journal apologise for an error in the above paper, which appeared in volume 25 part 3, pages 367-380. The error relates to the artwork of Fig. 6D on page 375, where the x-axis labels 'PanNET' and 'SINET' where transposed. The correct Fig. 6 is published in full below: 
A

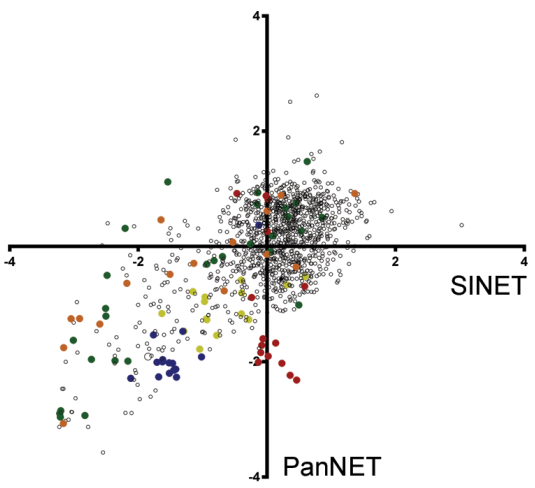

B

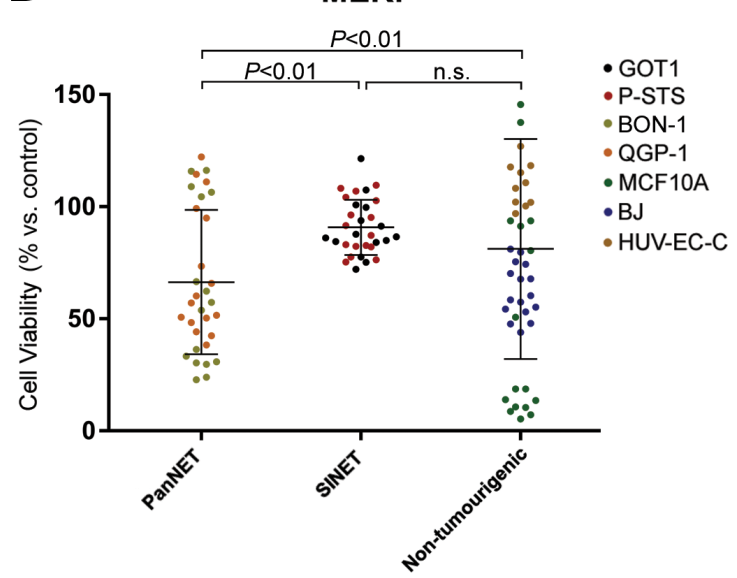

D

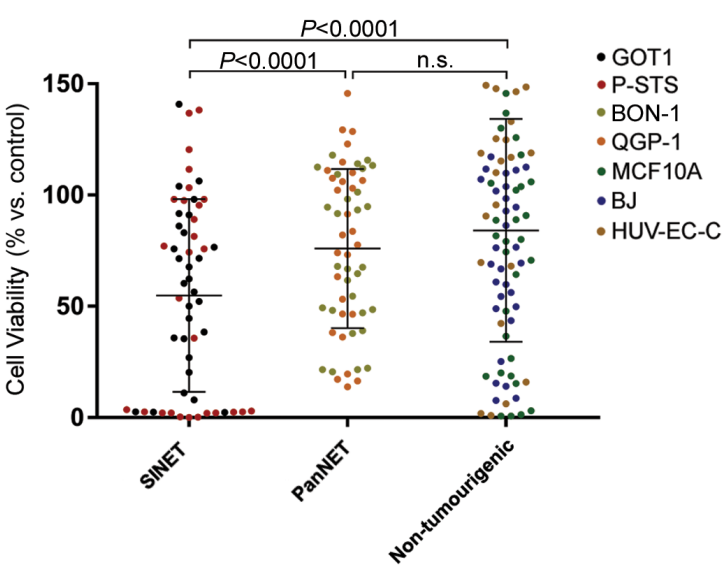

\begin{tabular}{|c|c|c|c|}
\hline \multicolumn{4}{|c|}{ PanNETs vs. SINETs } \\
\hline & Inhibitor target & Difference & Adj. $P$-value \\
\hline $\mathbf{\square}$ & HDAC & -1.23 & $6.4 \cdot 10^{-5}$ \\
\hline$\square$ & MEK & 1.08 & $1.9 \cdot 10^{-2}$ \\
\hline$\square$ & Topoisomeras & -0.99 & $9.6 \cdot 10^{-3}$ \\
\hline$\square$ & HSP90 & 0.94 & $1.7 \cdot 10^{-5}$ \\
\hline$\square$ & Aurora kinase & 0.54 & $2.3 \cdot 10^{-3}$ \\
\hline
\end{tabular}

C MEKi, primary culture

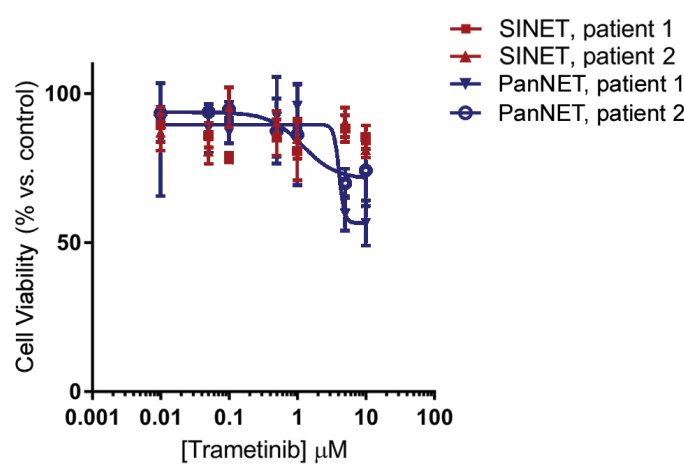

$E$

HDACi, primary culture

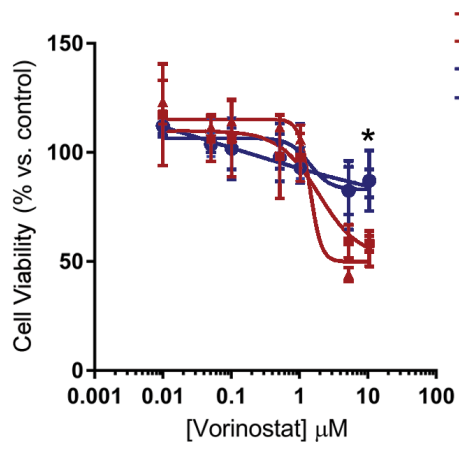

Figure 6

Therapeutic sensitivity of GEPNET cell lines and primary cell cultures. (A) Average Z-score representing the effect on cell viability of individual inhibitors to SINETs (GOT1/P-STS) and PanNETs (BON-1/QGP-1), plotted against each other. Groups of inhibitors that are significantly more potent against SINETs or PanNETs are marked by colour. (B) The effect of all MEKi against SINET cells, PanNET cells and non-tumourigenic cells. MEKi are more potent against PanNET cells, compared to SINET and non-tumourigenic cells. (C) Comparing the sensitivity of PanNET and SINET first-passage primary cells to MEKi trametinib. (D) SINET cell lines are more sensitive to HDACi, compared to PanNET cells and non-tumourigenic cells. (E) First-passage primary SINET cells are seemingly more sensitive than primary PanNET cells to the HDACi vorinostat. (B and D) Bars indicate mean effect, error bars S.D. and $P$ values generated from Wilcoxon signed-rank test. (C and E) Dose-response curves represent a mean of $n=3$ and the error bars denote standard deviation (s.D.). 